\title{
Convergence through divergence: compensatory changes in phonetic accommodation
}

\author{
Jevon Heath ${ }^{*}$
}

LSA Annual Meeting, Portland, January 8-11, 2015

Introduction. Phonetic accommodation is the phenomenon in which talkers alter subphonemic features of their speech in response to specific features of received speech stimuli. Convergence - talking more like an interlocutor - has generally been treated as the default form of accommodation (cf. Bourhis \& Giles 1977, Babel 2012, inter alia). This is presumably due to its ubiquity: convergence has been demonstrated to occur in natural conversation (Pardo 2006), as well as in response to both natural and manipulated recorded stimuli (Goldinger 1998; Nielsen 2011). In contrast, divergence is only attested as moderated or intentional alteration of speech (cf. Bourhis \& Giles 1977). This framing of convergence as the prototypical manifestation of phonetic accommodation implicitly rules out the possibility of accommodation leading to new phonetic variation within a speech community: movement from any point within a range of variation toward any other point within that range will necessarily end up also within that existing range.

However, most previous accommodation studies measuring phonetic features have looked at only one feature at a time. When considering multiple features at the same time, a greater range of accommodation behaviors is possible, including simultaneous convergence and divergence in different cues (antagonistic accommodation).

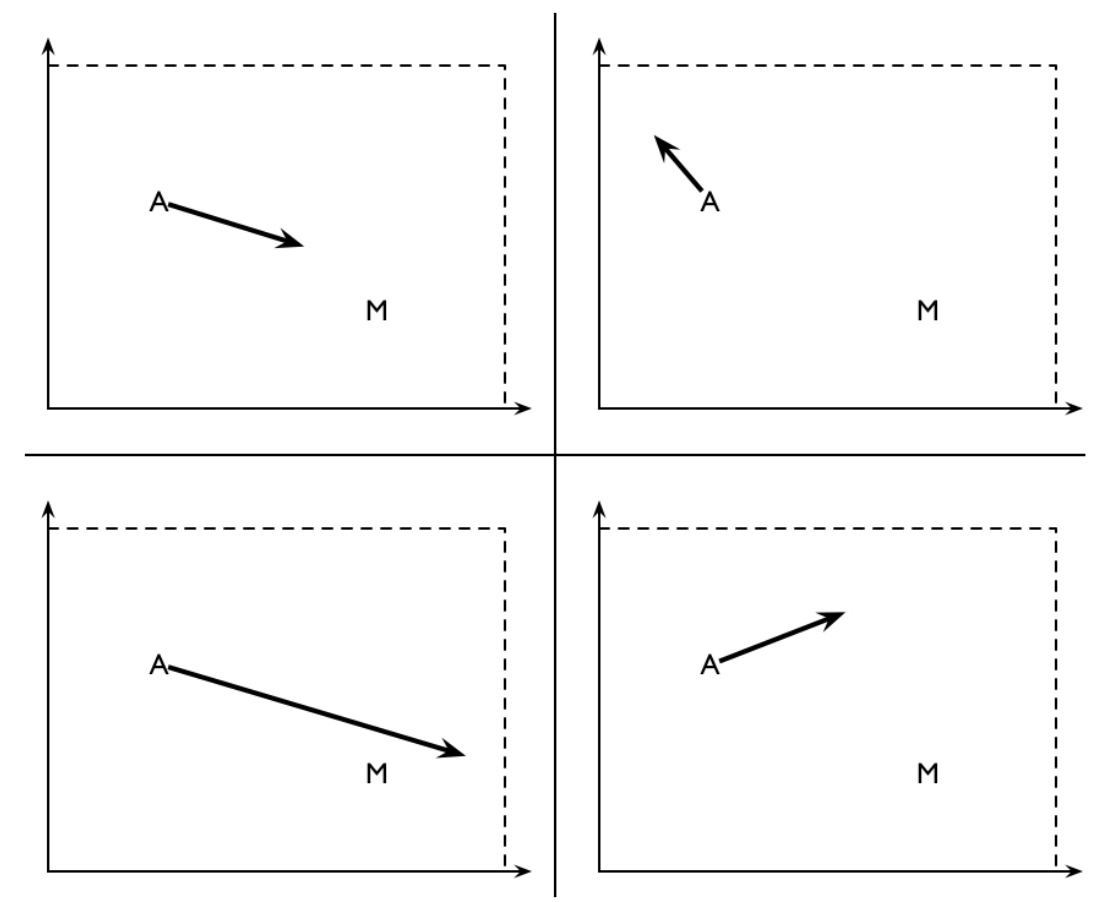

Figure 1. Accommodation to multiple features. $\mathrm{A}=$ accommodator, $\mathrm{M}=$ model. Clockwise from top left are: convergence; divergence; antagonistic accommodation; and hyperconvergence.

\footnotetext{
*I would like to thank Melinda Fricke, Sharon Inkelas, Christine Jiang, Keith Johnson, Susan Lin, Jeff Mielke, Julia Morse, Kuniko Nielsen, and Alan Yu, among others. Author: Jevon Heath, University of California, Berkeley (jevon.heath@berkeley.edu).
} 
While independent phonetic features may plausibly be adjusted in any of the ways illustrated in Figure 1, physiological or learned restrictions on articulation (cf. Kingston \& Diehl 1994) may mean that simultaneous convergence along incompatible dimensions is not always possible. This is schematized in Figure 2. The $x$ - and $y$-axes represent two phonetic features in an inverse relationship: $x$ increases as $y$ decreases, and vice versa, such that the speaker can only produce values along the diagonal line. The shaded area is the community's range of variation for these features. Given a model that the speaker is unable to produce faithfully, the closest approximation that the speaker can produce results in divergence along the $y$-axis and a variant outside the extant range of variation.

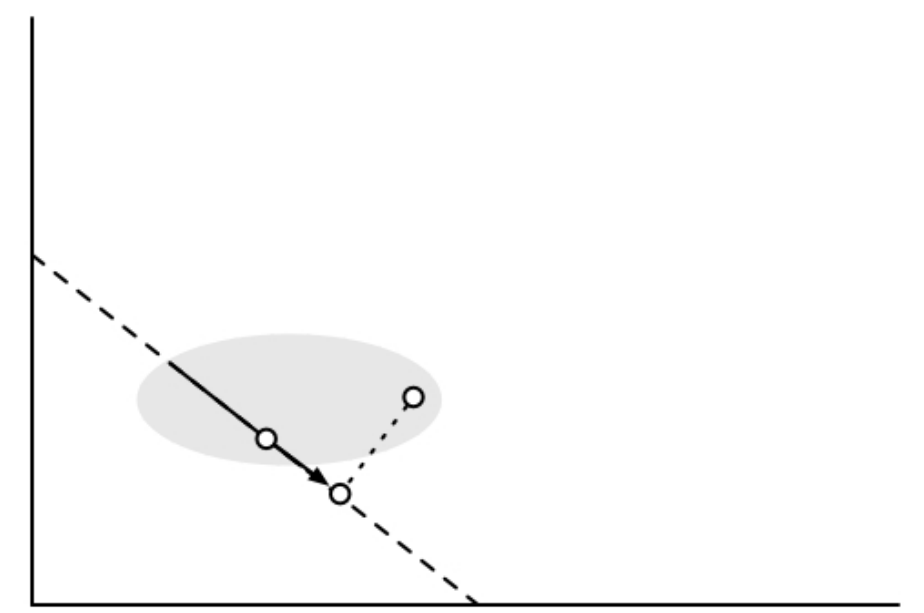

Figure 2. Accommodation leading to innovated variation

Of course, multiple potential accommodation strategies are available, such that the closest global approximation schematized in Figure 2 may not be the strategy used by all speakers. For example, since stop closure duration correlates inversely with voice onset time (VOT) (Lehiste 1970, Boucher 2002), speakers exposed to a model talker with extended VOT in their voiceless stops have several plausible ways of converging toward the model's speech. Speakers might target the model's lengthened VOT directly; or they might target the shorter closure-stop ratio (CSR) evinced by the model, either by increasing their VOT or by decreasing their closure duration; or else they might target the lengthened duration of the stop overall, either by increasing their VOT or their closure duration. Table 1 shows these possible targets of accommodation along with the behavior that would result. While some patterns of behavior would result from multiple strategies, it is the case that different strategies would result in each of VOT and stop closure duration variously lengthening and shortening.

\begin{tabular}{lll} 
Target & Intended adjustment & Coincident adjustment \\
\hline VOT & Increase VOT & Decrease closure \\
CSR & Increase VOT & Decrease closure \\
CSR & Decrease closure & Increase VOT \\
Stop duration & Increase VOT & Decrease closure \\
Stop duration & Increase closure & Decrease VOT
\end{tabular}

Table 1. Possible adjustments toward model with long VOT and average closure duration. 
In the current study, participants were exposed to the speech of just such a talker. While almost all participants converged significantly toward the model talker in at least one measured phonetic feature, over half of participants also diverged significantly from the model talker in at least one regard. I interpret these adjustments as compensatory changes resulting from individual learned patterns of articulation.

Methodology. The experiment consisted of an initial questionnaire, followed by a pre-exposure baseline recording, a shadowing task, and a post-exposure recording. In the baseline recording, participants read aloud a randomized list of 120 words. In the shadowing task, participants shadowed recordings of 80 words presented one at a time (twice each). Target words consisted of English words with a stressed second syllable beginning with one of /ptk/ (e.g., opposable, botanical). The VOT of the voiceless stops was artificially doubled; no other adjustment was made to the stimuli. The procedure and word list for the post-exposure recording was the same as for the pre-exposure recording. Only female monolingual English-speaking participants who gave permission to use their data in all contexts were considered for this study $(n=14)$. VOTs and closure durations from the pre-exposure and post-exposure blocks were hand-measured using Praat (Boersma \& Weenink 2014).

Findings. Results across participants are shown in Figure 2. Overall, participants converged in VOT $(M=4.758 \mathrm{~ms}, p<0.0001)$ across conditions, but diverged in stop closure duration $(M=$ $3.32 \mathrm{~ms}, p<0.0001)$. However, the closure-stop ratio converged significantly across participants $(M=2.82 \%, p<0.0001)$. Additionally, mean total stop duration across all participants did not differ significantly across conditions $(M=1.43 \mathrm{~ms}, p=0.158)$. These findings confirm the inverse correlation between VOT and stop closure duration, and suggest that it is the ratio of these two features that is the global target of accommodation, rather than VOT in itself.

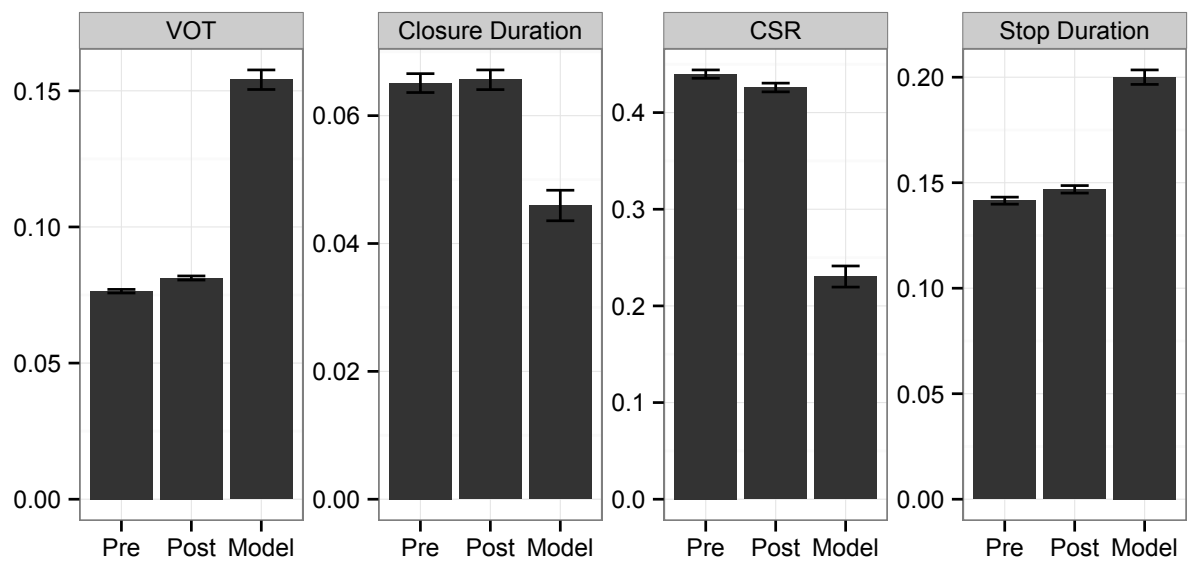

Figure 2. Global changes across conditions.

Results by participant are shown in Table 2. Despite the fact that VOT was the manipulated feature, only seven participants converged significantly toward the model in VOT. Exactly half of participants converged in stop closure duration, while half diverged. Ten participants converged in CSR; all of those who did not converged in closure duration, VOT, or both. Only two participants (S39 and S42) did not evince divergence in any feature. Only three participants (S17, S20, and S23) did not show significant adjustment of any feature. 


\begin{tabular}{|c|c|c|c|c|c|c|c|c|}
\hline & \multicolumn{2}{|c|}{ VOT (ms) } & \multicolumn{2}{|c|}{ Closure (ms) } & \multicolumn{2}{|c|}{ CSR (\%) } & \multicolumn{2}{|c|}{ Stop duration $(\mathrm{ms})$} \\
\hline Speaker & Pre & Post & Pre & Post & Pre & Post & Pre & Post \\
\hline Model & \multicolumn{2}{|c|}{$139.15 \mathrm{~ms}$} & \multicolumn{2}{|c|}{$61.48 \mathrm{~ms}$} & \multicolumn{2}{|c|}{$30.34 \%$} & \multicolumn{2}{|c|}{$200.63 \mathrm{~ms}$} \\
\hline S15 & 72.47 & $85.45 * * *$ & 56.55 & $47.78 * *$ & 43.88 & $35.29 * * *$ & 129.02 & 133.23 \\
\hline S17 & 60.62 & 64.10 & 52.21 & 50.62 & 46.44 & 44.40 & 112.83 & 114.73 \\
\hline S20 & 62.43 & 62.91 & 49.72 & 55.20 & 43.87 & 45.87 & 112.15 & 118.11 \\
\hline S21 & 79.41 & $88.89 * *$ & 55.05 & $43.52 * *$ & 39.63 & $32.82 * *$ & 134.46 & 132.41 \\
\hline S23 & 66.31 & 66.60 & 69.65 & 63.89 & 50.20 & 48.72 & 135.96 & 130.50 \\
\hline S24 & 67.83 & 68.15 & 54.37 & $45.86 * *$ & 43.74 & 40.20 & 122.19 & $114.00 *$ \\
\hline S25 & 77.13 & $82.47 *$ & 61.81 & $69.18 * * *$ & 44.42 & $45.71 *$ & 138.94 & $151.65 * * *$ \\
\hline S28 & 68.67 & 67.88 & 51.13 & $62.29 * * *$ & 43.19 & $48.60 * *$ & 119.80 & $130.17 * *$ \\
\hline S30 & 78.67 & 76.73 & 44.49 & $36.48 * *$ & 35.55 & 32.63 & 123.16 & $113.20 * *$ \\
\hline S32 & 65.12 & $69.15 *$ & 60.23 & $53.63 * *$ & 47.78 & $43.61 *$ & 125.35 & 122.78 \\
\hline S35 & 83.27 & $93.55 * * *$ & 66.67 & $49.73 * * *$ & 44.43 & $34.94 * * *$ & 149.94 & $143.29 *$ \\
\hline S39 & 69.78 & $83.69 * * *$ & 69.83 & $58.30 * * *$ & 50.00 & $40.75 * * *$ & 139.60 & 141.99 \\
\hline S40 & 76.25 & 79.42 & 43.51 & 48.85 & 36.39 & 38.03 & 119.76 & $128.26 * *$ \\
\hline S42 & 50.89 & $56.97 * *$ & 58.76 & 61.37 & 54.20 & 51.94 & 109.65 & $118.34 *$ \\
\hline
\end{tabular}

Table 2. Individual changes across conditions. Gray $=$ divergence; boxes $=$ hyperconvergence. Asterisks indicate significance levels of t-tests across conditions after Bonferroni correction.

$$
* p<0.05, * * p<0.01,{ }^{* * *} p<0.005 \text {. }
$$

Conclusion. These results indicate that divergence is an inevitable byproduct of accommodation in some circumstances. Despite population-wide convergence toward the experimentally manipulated phonetic variable, individual speakers had different targets in phonetic accommodation. Some speakers converged toward the model talker's VOT, some toward the model's closure duration, and some toward the ratio of the two features. The occurrence of divergence indicates that accommodation is a potential pathway for sound change; individual differences in the target of phonetic accommodation may reflect constraints on the potential pathways of sound change. Additionally, these findings complicate research on accommodation: since speakers may converge or diverge in other dimensions than those being measured, studies must take multiple dimensions of phonetic similarity into account in assessing the degree of accommodation speakers evince.

\section{References}

Babel, Molly. 2012. Evidence for phonetic and social selectivity in spontaneous phonetic imitation. Journal of Phonetics 40(1). 177-189.

Boersma, Paul \& David Weenink. 2014. Praat: doing phonetics by computer. Version 5.3.62, http://www.praat.org/. (2 January, 2014.)

Boucher, Victor J. 2002. Timing relations in speech and the identification of voice-onset times: A stable perceptual boundary for voicing categories across speaking rates. Perception \& Psychophysics 64 (1). 121-130.

Bourhis, Richard Y. \& Howard Giles. 1977. The language of intergroup distinctiveness. In Howard Giles (ed.), Language, ethnicity, and intergroup relations. New York: Academic Press.

Goldinger, Stephen D. 1998. Echoes of Echoes? An episodic theory of lexical access. Psychological Review 105(2). 251-279.

Kingston, John, \& Randy L Diehl. 1994. Phonetic knowledge. Language 70(3). 419-454.

Lehiste, Ilse. 1970. Suprasegmentals. Cambridge: Cambridge University Press.

Nielsen, Kuniko. 2011. Specificity and abstractness of VOT imitation. Journal of Phonetics 39(2). 132-142.

Pardo, Jennifer S. 2006. On phonetic convergence during conversational interaction. The Journal of the Acoustical Society of America 119(4). 2382-2393. 